\title{
Corona-Eindämmung in Taiwan
}

\section{Nur digitale Tools?}

\author{
Taiwan war mehr als andere Länder durch die Corona-Pandemie gefährdet, konnte die \\ Pandemie aber erfolgreich eindämmen. Digitale Tools spielten dabei eine wichtige, \\ aber keine ausschließliche Rolle. Doch wie sieht es mit dem Datenschutz aus?
}

Die Eindämmung der Corona-Pandemie in Taiwan ist bemerkenswert. Das Land konnte bislang eine größere Infektionswelle und umfassende Lockdowns vermeiden. Bis heute (3. Oktober 2020) zählt Taiwan 517 Infizierte mit 7 Toten (Deutschland: 298.475 Fälle, 9596 Tote). Dabei galt Taiwan anfangs als eines der gefährdetsten Länder, aufgrund der engen wirtschaftlichen und zwischenmenschlichen Beziehungen sowie geographischer Nähe zu China. Eine umfassende Darstellung von Taiwans Pandemiebekämpfung würde den Rahmen dieses Artikels sprengen. Stattdessen soll hier nur Taiwans Einsatz digitaler Tools besprochen werden. Der Blick auf Taiwan lohnt dabei aus mehreren Gründen. Zum einen ist das Land hochgradig digitalisiert. Fast $80 \%$ der Bevölkerung nutzen Smartphones, ${ }^{1}$ Breitbandzugang gilt als Menschenrecht ${ }^{2}$ und taiwanesische Firmen haben Schlüsselrollen in globalen IKT-Wertschöpfungsketten inne. Zum anderen ist Taiwan eine gefestigte liberale, rechtsstaatliche Demokratie mit starker Zivilgesellschaft. Im jährlichen Demokratie- und Freiheitsranking durch die amerikanische NGO Freedom House kommt Taiwan auf 93 von 100 Punkten (2019), vergleichbar mit Deutschland (94 von 100). ${ }^{3}$

\section{Früherkennung durch Social-Media}

Nach dem Schock der SARS-Epidemie 2003, die Taiwan unvorbereitet traf und 73 Tote kostete, überholte die Insel ihre Struk-

\footnotetext{
* Die diesem Aufsatz zu Grunde liegende Arbeit wurden mit Mitteln des Bundesministeriums für Bildung und Forschung unter dem Förderkennzeichen 16KIS0741K gefördert. Eine Vorgängerversion erschien am 04.05.2020 auf dem Corona-Tracing Blog des Forum Privatheit (https://corona.forum-privatheit.de/)

1 Statista 2020, "Number of smartphone users in Taiwan".

2 Republic of China [Taiwan], Executive Yuan 21.03.2017, "Executive Yuan unveils digital infrastructure project".

3 Freedom House 2020, „Freedom in the World 2020: Taiwan“, https://freedomhouse.org/country/taiwan/freedom-world/2020; idem., "Germany", https:// freedomhouse.org/country/germany/freedom-world/2020.
}

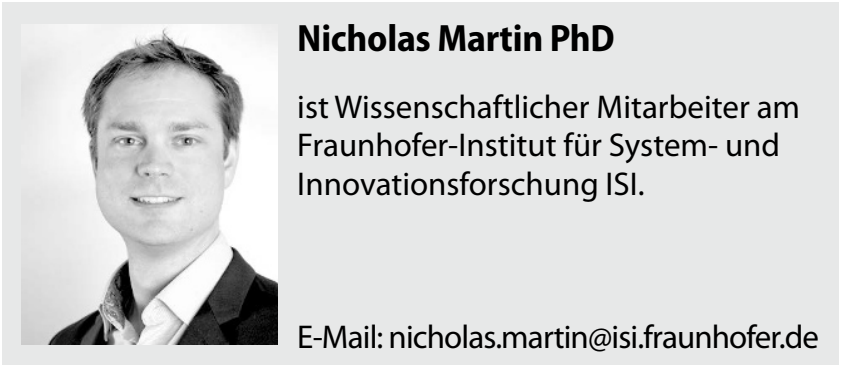

turen zur Pandemiebekämpfung grundlegend. Unter anderem wurde ein Frühwarnsystem aufgebaut, das Krankenhaus-, Medienberichte und Soziale Medien kontinuierlich auf Hinweise zu neuen Pandemien monitort. Social Media Postings chinesischer Ärzte aus Wuhan, die vor einer neuen Lungenkrankheit warnten, wurden so bereits am 31. Dezember 2019 erkannt und Eindämmungsmaßnahmen sofort eingeleitet. Chinesische Behörden wurden mit Bitte um Aufklärung kontaktiert und Einreisende aus Wuhan noch am gleichen Abend auf Fieber getestet und hospitalisiert. $^{4}$

\section{Contact-Tracing - ohne Tracing-App}

Taiwan hat beträchtliche, auch digitale Mittel in die Rückverfolgung von Infektionsketten (contact tracing) investiert, aber keine Tracing-App eingesetzt. (Im Gegensatz dazu veröffentlichte Singapur schon im März seine TraceTogether-App.) Dank Tuberkulose-Bekämpfungsprogrammen verfügte Taiwan bei PandemieAusbruch über eine entwickelte contact tracing-Infrastruktur; der zusätzliche Nutzen einer App wurde als gering eingeschätzt. ${ }^{5}$

Contact tracing in Taiwan funktioniert stattdessen so, dass ausgebildetes Personal die mit Corona Infizierten interviewt, um ihre Aufenthaltsorte und Kontakte der letzten 14 Tage zu ermitteln. Um contact tracer und Patienten - denen es oft schwerfällt, verlässliche Aussagen über mehr als die vergangenen ein bis zwei Tage zu treffen - zu unterstützen, wird regelmäßig eine Bewegungskarte für die Person auf Grundlage von Mobilfunkzellendaten erstellt. Bei Unklarheiten wurde gelegentlich auch auf CCTV-Aufnahmen zurückgegriffen. (Taiwan verfügt über ein relativ dichtes Netz von CCTV-Kameras. ${ }^{6}$ ) Als Rechtsgrundlage dient Art. 31 des taiwanesischen Infektionskrankheiten-Kontrollgesetz, ${ }^{7}$ gemäß dessen mit definierten Infektionskrankheiten Infizierte ihre Aufenthaltsorte und Kontakte medizinischem Personal gegenüber offenlegen müssen. Nach dem contact tracing werden die Daten in jedem Fall wieder gelöscht. ${ }^{8}$

4 Harvard Fairbank Center for Chinese Studies, "The Challenge of Covid-19: The Taiwan Experience", 2020, https://fairbank.fas.harvard.edu/events/webinarthe-challenge-of-covid-19-the-taiwan-experience; Wang/Ellis, "This Is How Taiwan Got a Head Start on Smashing the Virus", Bloomberg News 07.05.2020.

5 Telefoninterview, Dr. Anita Pei-chun Chan, Taiwan CDC, 25.04.2020.

6 Ricker, "The US, like China, has about one surveillance camera for every four people", The Verge, 02.12.2019.

7 RoC, "Communicable Disease Control Act", 2019.

8 Telefoninterview, Dr. Anita Pei-chun Chan, Taiwan CDC, 25.04.2020. 
In wenigen Fällen musste ausschließlich auf Funkzellenabfragen (ohne Interviews) und anschließende Massenkontaktierung per Mobilfunknachricht zurückgegriffen werden, wobei sich das als wenig effektiv erwies.

Taiwan versteht Digitaltechnik in erster Linie als Tool zur Unterstützung des medizinischen Personals, nicht als Ersatz für menschliche contact tracer (Interviewer). Das deckt sich auch mit singapurischem Denken. So betont der Entwickler von TraceTogether aus Singapur, dass die App menschliche contact tracer nie ersetzen, sondern nur unterstützen kann. ${ }^{9}$

\section{Zusammenführung von Datenbanken}

Taiwan begann bereits Anfang Januar internationale Risikogebiete auszuweisen, zuerst Wuhan, und dann nach und nach den Rest Chinas, andere asiatische Staaten, Europa und schließlich die übrige Welt. Alle Einreisenden aus Risikogebieten wurden (und werden) für 14 Tage unter Quarantäne gestellt (s.u.). Auch führte Taiwan die sonst getrennten Datenbanken der Einreisebehörde und der staatlichen Krankenversicherung zusammen, sodass Ärzte automatisch benachrichtigt werden konnten, falls sie von einem Patienten aufgesucht wurden, der in den vorherigen 14 Tagen aus einem Risikogebiet eingereist war. Tests und Schutzmaßnahmen für das medizinische Personal und andere Patienten konnten dann sofort eingeleitet werden. So sollte der Krankheitsverbreitung durch Infizierte vorgebeugt werden, die Taiwan betreten hatten, bevor ihr Ausreiseland zum Risikogebiet erklärt wurde. Ein weiteres Anliegen war, Ärzte und Pfleger möglichst gut vor Corona zu schützen, da sie zu den von SARS am schwersten Betroffenen gezählt hatten. Zudem konnten kürzlich aufgetretene Krankheitsfälle so erneut analysiert werden, um eventuell unerkannte gebliebene Corona-Fälle zu identifizieren..$^{10}$

Grundsätzlich wären derartige Datenzusammenführungen in Taiwan ohne richterliche Anordnung oder weitere Rechtsgrundlage illegal; das Infektionskrankheiten-Kontrollgesetz schafft Letztere jedoch. Aus Datenschutzgründen werden nur die Daten der letzten 14 Tage zusammengeführt. Die Datenbanken sollen nach der Pandemie wieder getrennt werden. ${ }^{11}$

Diese Zusammenführung war auch deshalb möglich, weil Taiwans Gesundheitssystem als Bürgerversicherung (single payer) organisiert ist, der mehr als 99\% der Taiwanesen und die meisten ausländischen Einwohner angehören. Das Gesundheitssystem ist bereits relativ digitalisiert, mit integrierten Datenbanken und digitaler Patientenakte. Da Gesundheitsdienstleister ihre Rechnungen der staatliche Versicherung innerhalb von 24 Stunden stellen müssen, verfügt deren Datenbank über Fast-Echtzeitinformationen über alle Arztbesuche und Behandlungen. ${ }^{12}$

9 Bay, "Automated contact tracing is not a coronavirus panacea", Medium, 11.04.2020, https://blog.gds-gov.tech/

automated-contact-tracing-is-not-a-coronavirus-panacea-57fb3ce61d98

10 Telefoninterview, Dr. Anita Pei-chun Chan, Taiwan CDC, 25.04.2020; Wang/ Ng/Brook , Response to COVID-19 in Taiwan: Big Data Analytics, New Technolo-

gy, and Proactive Testing", JAMA 323 (14), 2020, pp. 1341-1342.; Harvard Fairbank Center

11 Telefoninterview, T.H. Schee (taiw. Netzpolitik-Aktivist und Internet-Unternehmer), 17.04.2020; Wang/Ng/Brook 2020

12 Scott, "Taiwan's single-payer success story - and its lessons for America", Vox Media, 13.01.2020; Lin/Braund u.a., Policy Decisions and Use of Information Technology to Fight COVID-19 in Taiwan, Emerg Infect Dis. 26(7), 2020, pp.1506-1512.

\section{Digitale Überwachung von Quarantäne}

Corona-Verdachtsfälle und alle aus Risikogebieten Eingereiste müssen in Taiwan für 14 Tage in Quarantäne. Während dieser Zeit erhalten sie ein Tagesgeld von ca. €30; umgekehrt kann ein Bruch der Quarantäne mit Bußgeldern von bis zu rund $€ 30.000$ geahndet werden, wobei es scheint, dass man hier eine gewisse Milde hat walten lassen.

Die Einhaltung der Quarantäne wird digital überwacht. Unter Quarantäne stehende Personen müssen den örtlichen Gesundheits- und Polizeiämtern ihre Handy-Nummern übermitteln und dürfen das Gerät nicht ausschalten. Alternativ können sie sich auch ein Telefon geben lassen, wenn sie ihre Nummer nicht herausgeben möchten. Über Funkzellenortung wird dann ermittelt, ob das Handy sich am Quarantäne-Ort befindet. Das Gesundheitsamt ruft auch mehrmals täglich an, sowohl um sicherzustellen, dass die quarantänisierte Person sich in der Nähe ihres Handys befindet als auch um nach ihrem psychischen Wohlbefinden in der Isolation zu schauen. Wird der Quarantäneort laut Ortungssignal verlassen, werden Ermahnungen per SMS verschickt oder Telefonanrufe getätigt. Sofern die Person sich sofort an den Quarantäneort zurückbegibt, kommt es zu keiner Weiterverfolgung oder Bußgeldern; meldet das Ortungssignal aber eine andauernde Nichteinhaltung der Quarantäne, wird dies vor Ort durch Gesundheitspersonal und/oder Polizei geprüft. ${ }^{13}$

Dabei scheinen Fehlalarme aufgrund von Mobilfunklücken, leeren Handy-Batterien u.Ä. relativ häufig vorgekommen zu sein und zu einiger gefühlten Belästigung (aber keinen Bußgeldern o.Ä.) geführt zu haben. So kam es laut eines taiwanesischen Digitalaktivisten in der Stadt Taoyuan innerhalb von zwei Tagen zu 740 Fehlalarmen - von 741 Alarmen insgesamt! $!^{14}$

Dieser sog. „digitale Zaun“ wurde vom Netzbetreiber Chunghwa Telecom in Kooperation mit der Regierung entwickelt und betrieben. Die erhobenen Daten sollen nur den jeweils zuständigen lokalen Behörden vorliegen und müssen nach der 14-tägigen Quarantäne sofort gelöscht werden. Zugriff auf das Gesamtsystem haben laut Medienberichten nur „der Gesundheitsminister“ (?) sowie drei Mitarbeiter von Chungwha. Sobald die Pandemie abklingt, sollen das System und alle noch vorliegenden Daten gelöscht und über Audits sichergestellt werden, dass keine Daten illegal zurückgehalten werden. ${ }^{15}$

\section{Aggregierte Erfassung von Massen-bewegungen}

Um Menschenaufläufe, von denen eine erhöhte Ansteckungsgefahr ausgehen könnte, zu verhindern, verfolgte Taiwan Massenbewegungen zu Ferientagen mittels aggregierter Funkzellendaten. Beim Auftreten von zu großen oder dichten Menschenansammlungen wurden Nachrichten an die in den jeweiligen Funkmasten eingeloggten Handys verschickt, mit der Bitte, social dis-

13 Telefoninterview, T.H. Schee, 17.04.2020; Hsieh, „Coronavirus: Under surveillance and confined at home in Taiwan", BBC, 24.03.2020; Donovan Smith, "Quarantined: When Taiwan's Phone Tracking System Goes Awry", Ketalang Media 03.04.2020; Harvard Fairbank Center

14 Telefoninterview, T.H. Schee, 17.04.2020; Hsieh 2020; Donovan Smith 2020.

15 Telefoninterview, T.H. Schee, 17.04.2020; Sander, „B, Bewegung nur noch in einem 50-Meter-Radius erlaubt: wie Taiwans Handy-Überwachung funktioniert", NZZ 19.04.2020. 
tancing zu befolgen und nach Möglichkeit die Ansammlung zu verlassen. Hierzu nutzte das Land bestehende Systeme zur Verschickung von Warnhinweisen und Informationen an die Bevölkerung im Katastrophenfall. Diese existieren schon länger, aufgrund der hohen Taifun- und Erdbebengefahr in Taiwan und der militärischen Bedrohung durch China. ${ }^{16}$

\section{Einbindung der Zivilgesellschaft: Maskenzugang und Bekämpfung von Fake News}

In Taiwan wie in vielen ostasiatischen Ländern ist das Tragen von Gesichtsmasken zum Schutz vor Infektionskrankheiten mindestens seit der SARS Epidemie übliche gesellschaftliche Praxis. Entsprechend bald kam es nach Bekanntwerden der Corona-Pandemie zu Panik- und Hamsterkäufen von Masken, die die allgemeine Versorgung der Bevölkerung und des Gesundheitssystems mit Masken bedrohten. In zumindest einem Fall wurden diese durch vorsätzlich gestreute Gerüchte befeuert. Um die Versorgung aller mit Masken sicherzustellen und Preistreiberei zu verhindern, wurden diese daraufhin temporär rationiert (bis größere Produktionskapazitäten aufgebaut werden konnten). Maskenkauf (zu staatlich festgelegten Niedrigpreisen) war fortan nur mit der digitalen Krankenversicherungskarte möglich (die alle Versicherten, d.h. mehr als 99\% der Bevölkerung besitzen). Karteninhaber hatten Anspruch auf 3 Masken/Woche. Gleichzeitig wurde Echtzeitdaten über Maskenbestände und -verfügbarkeiten in allen Apotheken des Verteilsystems öffentlich gemacht, so dass civic hacker aus der Zivilgesellschaft Karten und andere Apps, die über Maskenverfügbarkeit informierten, entwickeln konnten. Tatsächlich wurden bald über 100 Apps gebaut. ${ }^{17}$

Allgemeiner stellte sich auch in Taiwan die Frage, wie in der angespannten Situation die Ausbreitung von Gerüchten und Ängsten in der Bevölkerung verhindert werden sollte. Diese Frage war besonders akut, da Taiwan seit längerem von China ausgehenden, aggressiven Desinformationskampagnen ausgesetzt ist. ${ }^{18}$ Sowohl analoge wie digitale Maßnahmen wurden eingeleitet.

Auf der analogen Seite gaben der Gesundheitsminister, der Vizepremier (ein bekannter Virologe) und die Leitung des Epidemie-Kontrollamts während der Hochzeit der Pandemie in Taiwan tägliche Presskonferenzen. Auf der digitalen Seite wurde das bereits 2019 eingeführte „2-2-2“-System verstärkt. Als Teil dieses Systems wurde in jedem Ministerium eine dedizierte Einheit mit der Aufgabe geschaffen, im Internet aufkommende Falschinformationen und Gerüchte zu identifizieren und im Schnellverfahren zu entkräften. Gegendarstellungen sind innerhalb einer Stunde nach Bekanntwerden der Falschinformation zu veröffentlichen, sollen nicht mehr als 20 Schriftzeichen im Titel und 200 Schriftzeichen in Volltext sowie mindestens 2 Bilder enthal-

16 Lee, „Virus Outbreak: CECC messages warn against crowding", Taipei Times, 05.04.2020; Telefoninterview, T.H. Schee, 17.04.2020.

17 Fondation pour la Recherche Stratégique, „A conversation with Audrey Tang", 2020; Leonard, „How Taiwan's Unlikely Digital Minister Hacked the Pandemic", Wired, 23.07.2020.

18 MacAuliffe, "Countering China's Sharp Power: Disinformation and Social Media in Taiwan", George Washington University Sigur Center, 2019; Silverman, "Chinese Trolls Are Spreading Coronavirus Disinformation In Taiwan", Buzzfeed News, 05.03.2020. ten. ${ }^{19}$ Die Gegendarstellung soll möglichst witzig gestaltet und als meme verpackt werden, um die stärkstmögliche Viralität zu erziehlen. In einer solchen Videomontage wackelte sogar der Vizepremier mit dem Hintern, um Gerüchte über ToilettenpapierKnappheit in Folge von Maskenproduktion zu entkräften. (Im tatsächlich von einem Toilettenpapierhändler gestreuten - Gerücht hatte es geheißen, dass Masken und Papier aus dem gleichen Material gefertigt würden, und das Papier daher knapp werde..$^{20}$ )

Als Teil dieser Strategie werden zivilgesellschaftliche Kräfte - insbesondere die in Taiwan seit einigen Jahren bestehende CoFacts-Community sowie internationale fact-checker Netzwerke - systematisch eingebunden, sowohl um Informationen über aufkommende Gerüchte und Falschinformationen zu bekommen als auch um die Gegendarstellungen ins Netz zu tragen und viral gehen zu lassen. ${ }^{21}$

\section{Institutioneller, politischer und rechtlicher Kontext}

Taiwan konnte schnell und effektiv auf Corona reagieren, weil die Jahre nach der SARS-Epidemie genutzt wurden, um die erforderlichen institutionellen und rechtlichen Strukturen aufzubauen, um sehr schnell auf epi-/pandemische Infektionskrankheiten reagieren zu können. Zum einen wurde mit dem Central Epidemic Command Center eine Institution geschaffen, die im Epidemiefall die relevanten Aktivitäten der Ministerien und Behörden zentral koordinieren können würde. Zum anderen wurde das Infektionskrankheiten-Kontrollgesetz - die wichtigste Rechtsgrundlage für das Vorgehen im Ernstfall - angepasst, was wiederum zu Klagen und Entscheidungen des Verfassungsgerichts und weiteren Überarbeitungen des Gesetzes einschließlich zusätzlicher Absicherungen für Bürgerrechte führte. Somit existierte beim Ausbruch von Corona ein klarer und auf seine Verfassungskonformität geprüfter Rechtsrahmen. ${ }^{22}$ Physische Infrastruktur, wie etwa Fiebermessgeräte an den meisten öffentlichen und vielen privaten Gebäuden, konnte nach dem Ausbruch ebenfalls rasch eingerichtet werden.

Wichtig zur Einordnung von Taiwans Vorgehen ist aber auch der breitere politische Kontext. Die Pan-Green Coalition unter Führung der Democratic Progressive Party, die seit 2016 an der Regierung ist, hat ihre Wurzeln im Kampf gegen die KuomintangDiktatur in den 1970er und 1980er Jahren und versteht sich selbst als linksliberale Bewegung, deren ideologisches Fundament in Menschenrechten und Demokratie liegt. Sie unterhält relativ enge Beziehungen zu Menschenrechts- und zivilgesellschaftlichen Gruppen in Taiwan. Etwa war die (parteilose) Digitalministerin Audrey Tang bis zu ihrem Amtsantritt eine Netzaktivistin. Sie versteht das Internet und offene Daten als Mittel zur Demokratisierung von Staat und Gesellschaft, und zum Empowerment und zur Befähigung von zivilgesellschaftlichen Akteuren. Erfolgreiches Regieren, in ihrem Verständnis, beruht darauf die Kreativi-

19 Aufgrund der hohen Prägnanz der chinesischen Sprache enthalten 200 Zeichen meist mehr Information als 200 Wörter im Deutschen.

20 Everington, ,Rumors over face mask shortage spark run on toilet paper in Taiwan: Update", Taiwan News, 10.02.2020

21 Leonard 2020; Tang, "Finding Facts in a World of Disinformation”, 25.09.2019, https://www.youtube.com/watch?v=10uR4_dctTg\&t=143s.

22 Chang, "Taiwan's Fight against COVID-19: Constitutionalism, Laws, and the Global Pandemic", Verfassungsblog.de, 21.03.2020. 
tät und Intelligenz der Zivilgesellschaft zu stimulieren und einzubinden. ${ }^{23}$ Sorgen, dass die Pandemie zum Aufbau bleibender staatlicher Überwachung missbraucht werden könnte, waren in diesem Kontext weniger realistisch. Tatsächlich organisierten Abgeordnete der Regierungsparteien selbst parlamentarische Anhörungen, um Datenschutz während der Pandemie zu diskutieren. ${ }^{24}$

\section{Implikationen}

Die Mär vom „gehorsamen Asiaten“ oder „konfuzianischem Kollektivismus“ bietet keine Hilfe für die Einordnung von Taiwans Eindämmungsstrategie. Das Land ist eine streitbare Demokratie mit aktiver Zivilgesellschaft und starken Protestbewegungen. ${ }^{25}$ Seine Strategie ist also als eine mögliche, überlegte Reaktion einer reifen Demokratie, die auf Augenhöhe mit der unseren steht, zu betrachten. Welche Implikationen hat sie damit für die deutsche Debatte?

Es sticht heraus, dass Taiwan Digitaltechnik umfangreich einsetzt, der Technikeinsatz aber zur Unterstützung, nicht dem Ersatz menschlicher Entscheidungen und menschengeleiteter Prozesse dient. Wo allein Technik zum Einsatz kam, waren die Ergebnisse anscheinend bislang eher weniger gut. Ebenso ist interessant, wie Taiwans Digitalstrategie oft auf der aktiven Einbindung der Zivilgesellschaft zu beruhen scheint, ohne aber eine Vereinnahmung dieser anzustreben. Die civic hacker bleiben unabhängig.

Taiwans Strategie wirft aber auch Fragen zur Abwägung von Rechten, Pflichten und den verschiedenen Dimensionen einzelner Grundrechte, auf. Verkürzt könnte man sagen, dass Taiwan entschieden hat, (Datenschutz-)Rechte Einzelner temporär erheblich - aber nicht vollständig - einzuschränken, um damit einen relativ effektiven Schutz des Rechts auf Leben der Mitmenschen

23 De Borchgrave, "How to handle Covid“, Tortoise Media, 03.06.2020.

24 Telefoninterview, Prof. Yachi Chiang, National Taipei University of Technology, 25.05.2020.

25 Beispielsweise besetzten Aktivisten der "Sonnenblumen-Bewegung" 2014 das Parlamentsgebäude für 24 Tage. Ho, „Occupy Congress in Taiwan: Political Opportunity, Threat, and the Sunflower Movement ", J. of East Asian Studies 15(1), 2015. der Infizierten und den Aufrechterhalt der Freiheitsrechte dieser Mitmenschen zu erkaufen. Infizierte mussten umfangreiche, elektronisch abgeprüfte Angaben zu ihren Aufenthaltsorten und Kontakten machen; die unter Quarantäne stehenden Personen mussten Handyortung zulassen; umfangreiche Datenverknüpfungen wurden vorgenommen. Dafür konnten umfassendere Lockdowns und damit einhergehende Eingriffe in die Grundrechte sowie in das Sozial- und Wirtschaftsleben weiter Teile der Bevölkerung umgangen werden. Digitalministerin Tang sprach in diesem Zusammenhang von ,tiefen, aber sehr engen und zeitbegrenzten" Rechtseingriffen. ${ }^{26}$

Allerdings ist auch dieser Eingriff in die Datenschutzrechte der Infizierten nicht absolut. Freiwilligkeit, das Recht, selbstständig über die eigenen Daten zu bestimmen, ist massiv beschnitten worden; andere Datenschutzprinzipien aber bleiben gewahrt. Die erzwungenen Datenverarbeitungen scheinen zweckgebunden unter Beibehalt des Prinzips der Nichtverkettung mit zweckfremden Daten erfolgt zu sein, eingehegt durch weitere Schutzgarantien wie kurze Speicherfristen, Audits und rasche Löschung.

Wie derartige Abwägungen zu treffen sind, ist letztlich eine politische und keine ausschließlich rechts-, sozial- oder technikwissenschaftliche Frage. Sie bedarf einer breiten gesellschaftlichen Debatte und nicht nur einer Diskussion unter Fachexperten, und muss auch vor dem Hintergrund der jeweiligen institutionellen und infrastrukturellen Gegebenheiten entschieden werden. Etwa stellt sich die Frage, ob Corona-Infizierten das Recht zugestanden werden soll, selbst zu bestimmen, ob sie (per App oder mit anderen Mitteln) Kontakte über die Ansteckung informieren, anders dar, wenn unentgeltliche Tests denen vorbehalten bleiben, die nachweislich Kontakt zu Infizierten hatten, als wenn sie für alle verfügbar sind. Ebenso kann die Frage, ob eher die allgemeine Versammlungsfreiheit oder die informationelle Selbstbestimmung Einzelner zu schützen ist, je nach politischem und institutionellem Kontext anders bewertet werden.

Taiwan hat die Jahre nach SARS für diese Diskussion genutzt. Bei uns beginnt sie erst.

26 Kim, „Audrey Tang on her "conservative-anarchist" vision for Taiwan's future", restofworld.org, 29.09.2020.

\section{Springer Gabler}
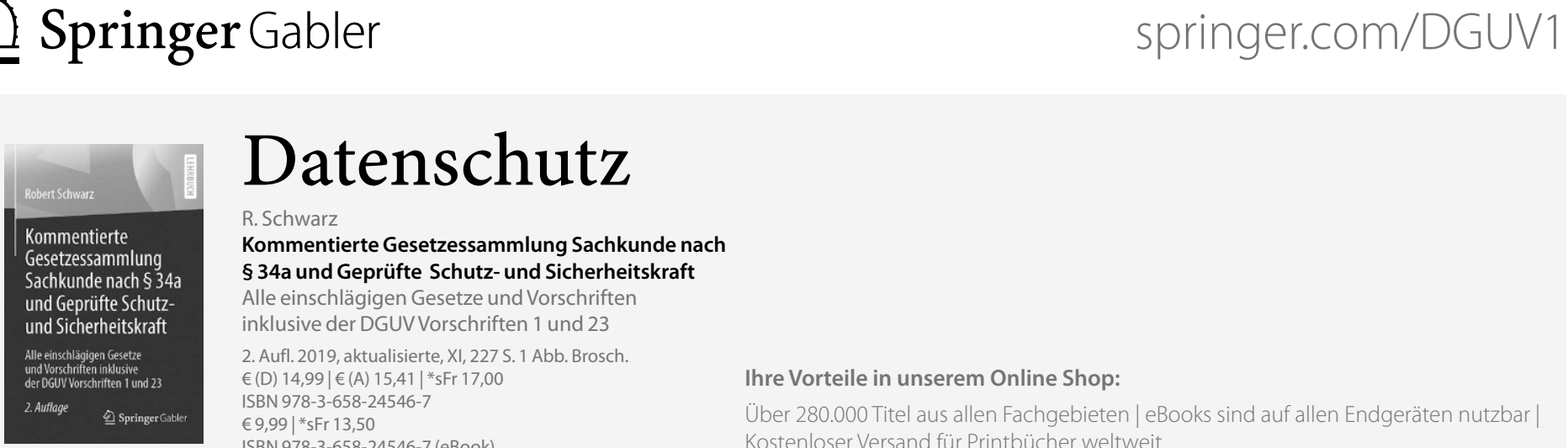

\section{Datenschutz}

R. Schwarz

Kommentierte Gesetzessammlung Sachkunde nach § 34a und Geprüfte Schutz- und Sicherheitskraft

Alle einschlägigen Gesetze und Vorschriften

inklusive der DGUV Vorschriften 1 und 23

2. Aufl. 2019, aktualisierte, $\mathrm{XI}, 227 \mathrm{~S} .1$ Abb. Brosch.

$€(D) 14,99|€(A) 15,41|^{*} \operatorname{sFr} 17,00$

ISBN $978-3-658-24546-7$

$€ 9,99 \mid{ }^{*} \mathrm{~s} F r$ 13,50

ISBN 978-3-658-24546-7 (eBook)
Ihre Vorteile in unserem Online Shop:

Über 280.000 Titel aus allen Fachgebieten | eBooks sind auf allen Endgeräten nutzbar Kostenloser Versand für Printbücher weltweit 